\title{
PREVALENCIA DE COMORBILIDADES EN PACIENTES CON ENDOMETRIOSIS EN EDAD FÉRTIL EN EL HOSPITAL MARÍA AUXILIADORA ENTRE LOS AÑOS 2008-2017
}

\author{
PREVALENCE OF COMORBIDITIES IN PATIENTS WITH ENDOMETRIOSIS OF CHILDBEARING \\ AGE IN THE MARÍA AUXILIADORA HOSPITAL BETWEEN 2008-2017
}

Pedro Infante-Bustamante1,2, Manuel J. Loayza-Alarico², Lucy E. Correa López ${ }^{3}$

\begin{abstract}
RESUMEN
Objetivo: Describir la prevalencia de comorbilidades en pacientes con endometriosis en edad fértil en el Hospital María Auxiliadora entre los años 2008-2017. Métodos: Se realizó un estudio cuantitativo, observacional y descriptivo. Se trabajó a partir de la base de datos de pacientes que se encontraban con diagnóstico de endometriosis en el Hospital María Auxiliadora. Resultados: Se encontraron 66 historias clínicas que contaban con el diagnóstico definitivo de endometriosis, de las cuales 60 se encontraban en edad fértil, el cual fue dividido en 3 grupos etarios, 20 a 40 años con 34 pacientes y 41 a 50 años con 26 pacientes. No se encontraron pacientes en el grupo de 11 a 19 años. La comorbilidad, en el grupo de edad fértil, con mayor prevalencia fue la anemia, con $81.7 \%(n=49)$, seguida de la enfermedad pélvica inflamatoria con $56.7 \%(n=34)$ y la miomatosis con $30 \%(n=18)$. La infertilidad se presentó en un $18.3 \%(n=11)$, la obesidad $10 \%(n=7)$ y las comorbilidades con una prevalencia menor al 10\%, fueron la ansiedad con $6.7 \%(n=4)$, la depresión, la hipertensión arterial y la patología coronaria con $5 \%(n=3)$, la diarrea, el colon irritable y la constipación se presentaron en menos del $5 \%$ de las pacientes y no se presentaron casos de cáncer de ovario, infarto de miocardio, ni linfoma no Hodking, pero sí en el grupo de mayores de 50 años, que presentó 1 caso de cáncer de ovario. El grupo etario que presentó mayor número de comorbilidades fue de 41 a 50 años con $51.9 \%(n=70)$. Conclusión: Se encontraron un gran número de casos de las comorbilidades estudiadas, a pesar de ser un grupo pequeño de pacientes, siendo el grupo de 41 a 50 años el más afectado. La alta prevalencia de anemia en nuestro país puede ser la razón de la cifra tan elevada en nuestras pacientes, a diferencia de la enfermedad inflamatoria pélvica que posee una prevalencia similar a la de otros estudios.
\end{abstract}

Palabras clave: Comorbilidad; Endometriosis; Prevalencia; Enfermedad inflamatoria pélvica; Miomatosis; Infertilidad. (fuente: DeCS BIREME)

\begin{abstract}
Objetive: To describe the prevalence of comorbidities in patients with endometriosis of childbearing age in the María Auxiliadora Hospital between 2008-2017. Methods: A quantitative, observational and descriptive study was carried out. We worked from the database of patients who were diagnosed with endometriosis at the María Auxiliadora Hospital. Results: We found 66 clinical histories that had the definitive diagnosis of endometriosis, of which 60 were of childbearing age, which was divided into 3 age groups, 20 to 40 years with 34 patients and 41 to 50 years with 26 patients. No patients were found in the group of 11 to 19 years. Comorbidity, in the fertile age group, with the highest prevalence was anemia, with $81.7 \%$ ( $n=$ 49), followed by pelvic inflammatory disease with $56.7 \%(n=34)$ and myomatosis with $30 \%(n=18)$. Infertility was presented in $18.3 \%(n=11)$, obesity $10 \%(n=7)$ and comorbidities with a prevalence lower than $10 \%$, were anxiety with $6.7 \%(n=4)$, depression, arterial hypertension and coronary disease with $5 \%(n=3)$, diarrhea, irritable bowel and constipation occurred in less than $5 \%$ of patients and there were no cases of ovarian cancer, myocardial infarction, or lymphoma not Hodking, but in the group of people over 50, who presented 1 case of ovarian cancer. The age group with the highest number of comorbidities was 41 to 50 years with $51.9 \%(n=70)$. Conclusion: A large number of cases of the comorbidities studied were found, despite being a small group of patients, with the group of 41 to 50 years being the most affected. The high prevalence of anemia in our country may be the reason for the high number in our patients, unlike the pelvic inflammatory disease that has a prevalence similar to that of other studies.
\end{abstract}

Key words: Comorbidity; Endometriosis; Prevalence; Pelvic inflammatory disease; Myomatosis; Infertility. (source: MeSH NLM)

\footnotetext{
${ }^{1}$ Hospital María Auxiliadora, Lima-Perú.

${ }^{2}$ Facultad de Medicina Humana, Universidad Ricardo Palma, Lima-Perú.

${ }^{3}$ Instituto de Investigación en ciencias biomédicas, Universidad Ricardo Palma, Lima-Perú.
}

Citar como: Pedro Infante-Bustamante, Manuel J. Loayza-Alarico, Lucy E. Correa López. Prevalencia de comorbilidades en pacientes con endometriosis en edad fértil en el Hospital María Auxiliadora entre los años 2008-2017. [Artículo Original].2018;18(4):53-58. (Octubre 2018). DOI 10.25176/RFMH.v18.n4.1731 


\section{INTRODUCCIÓN}

La endometriosis es definida como la implantación tanto de glándulas, como de estroma endometrial, fuera de la cavidad uterina. Tiene una prevalencia de aproximadamente el $10 \%$ de toda la población femenina, sin embargo, puede afectar alrededor del $20-90 \%$ de mujeres que padecen dolor pélvico e infertilidad ${ }^{1,2,3,4,5}$. En el Perú se estima que puede presentarse a partir de los 13 años, incluso en adolescentes que aún no han presentado la menarquia6.

La endometriosis es hormono-dependiente, por lo sus síntomas decaen con la menopausia ${ }^{2}$. La implantación de los focos determina muchas veces el grado de complicación de cada paciente. Las localizaciones más frecuentes suelen ser el peritoneo de la pelvis menor, trompas de Falopio, ovarios y ligamentos útero-sacro posteriores ${ }^{5}$ y entre las menos frecuentes las extrapélvicas, tales como pared abdominal ${ }^{7,8}$, tracto urinario ${ }^{9}$, tórax ${ }^{10,11}$, cicatrices perineales ${ }^{12}$, tracto gastrointestinal ${ }^{13,14} \mathrm{y}$ hasta incluso mucosa nasal ${ }^{5}$.

La causa de como los implantes endometriales pueden ubicarse fuera de la cavidad uterina son numerosas, dentro de las más conocidas están la menstruación retrógrada que consiste en la siembra ectópica de tejido endometrial descrita por Samson ${ }^{5,15,16}$, la diseminación linfática y vascular, la metaplasia celómica que consiste en la transformación del tejido celómico ubicado en la superficie del ovario, así como otros enfoques genéticos ${ }^{17,18}$, endocrinos ${ }^{19}$, inmunológicos ${ }^{20} \mathrm{e}$ incluso ambientales ${ }^{5}$.

Dentro de los principales síntomas causados por la endometriosis se encuentran la dismenorrea, la dispareunia y la disqueccia, y dentro de los principales signos están el sangrado intermenstrual y nódulos palpables en el fondo de saco posterior de la vagina si los implantes se encuentran en los ligamentos útero-sacros ${ }^{15}$.

El diagnóstico de la endometriosis es un verdadero reto, puesto que la clínica es muy variable, y el principal método consiste en realizar una biopsia del tejido endometrial en el sitio que creemos se encuentra. La laparoscopía, así como cualquier método quirúrgico que permita obtener ese tejido terminan siendo las técnicas de elección. Como se sabe al ser intervencionistas representan no sólo un alto costo, sino también todas las complicaciones asociadas a estas técnicas, razón por la cual se produce un retraso de entre 5 a 11 años ${ }^{21}$.
El tratamiento tiene que obedecer a tres pilares importantes: tratamiento temprano después de realizado el diagnóstico, tratamiento hormonal para suprimir o retrasar la progresión de la enfermedad y el manejo del dolor ${ }^{15}$.

Dentro de las comorbilidades asociadas a la endometriosis se encuentran:

La infertilidad: la tasa de fecundidad en una paciente con endometriosis se estima entre un $2 \%$ a un $10 \%$, muy por debajo de una mujer sana que es entre 15\% a $20 \%{ }^{22,23}$. Esto es causado debido a múltiples factores tales como las alteraciones anatómicas causadas por adherencias, la alteración de la función peritoneal por acumulación de líquido, alteración de la inmunidad y respuesta hormonal, disminución de la calidad del óvulo y del transporte tubo-ovárico ${ }^{22}$.

Cáncer: la endometriosis se asocia al cáncer de ovario y el linfoma no Hodking, aunque no existe evidencia de cómo los causa, pero se menciona que el $8 \%$ de las pacientes con endometriosis poseen una variante atípica fuertemente asociada al cáncer de ovario ${ }^{24}$.

Patología Gastrointestinal: dependiendo del sitio de implantación pueden presentarse cuadros de diarrea, constipación o hasta padecer colon irritable ${ }^{25}$.

Obesidad: se describe que la aromatasa producida por los adipocitos podría jugar un rol en los síntomas de la endometriosis, además se describe un aumento de peso en estas pacientes ${ }^{25,26}$.

Patología cardiovascular: el aumento del estrés oxidativo y de lipoproteínas de baja densidad producto de un estado inflamatorio crónico puede asociarse a un riesgo de 1.4 a 1.6 mayor de sufrir infarto agudo de miocardio, así como enfermedad coronaria ${ }^{25,27}$.

Miomatosis: por ser hormonodependientes pueden presentarse en simultáneo, además de poseer síntomas similares, aún no se ha demostrado una relación de causalidad ${ }^{25}$.

Enfermedad pélvica inflamatoria: existe un riesgo 1.1 mayor de padecerla si una mujer tiene endometriosis, incluso se puede presentar de forma más severa ${ }^{28,29}$.

Anemia: las pacientes con endometriosis tienen un riesgo 1.4 veces mayor de padecerla ${ }^{28}$

Ansiedad y Depresión: producto del efecto deletéreo sobre la calidad de vida de las pacientes con endometriosis, así como el padecimiento físico ${ }^{1,30,31}$. 


\section{MÉTODOS}

Se realizó un estudio cuantitativo, observacional y descriptivo. Se consideró para el estudio a todas las pacientes diagnosticadas con endometriosis entre las edades de 11 a 50 años, divididos en los grupos de 11 a 19 años, 20 a 40 años y 41 a 50 años, entre los años 2008-2017, en el Hospital María Auxiliadora. Técnica: revisión de historias clínicas del archivo del Hospital María Auxiliadora. Análisis de las Variables: Se realizó el análisis de datos en los programas Microsoft Office Excel 2016 y SPSS Statistics 25.0.

\section{RESULTADOS}

Se logró recolectar información de 66 pacientes con diagnóstico de endometriosis en el hospital María Auxiliadora, en el período 2008-2017, de los cuales se consideraron los que se encontraban dentro del período de edad fértil de la mujer, quedando 60 pacientes distribuidas de la siguiente manera: 34 (51.5\%) se encontraban en el grupo de 20 a 40 años y 26 (39.4\%) se encontraban en el grupo de 41 a 50 años, no se encontraron pacientes en el grupo de 11 a 19 años (Tabla 1).

La media de la edad del total de las pacientes diagnosticadas con endometriosis fue de $39.3+-9.6$ años (IC 95\%) (Tabla 1).

Entre las pacientes diagnosticadas con endometriosis, el $30.3 \%(n=20)$ presentaban 3 comorbilidades, el $27.3 \%(n=18)$, presentaban 1 comorbilidad, el $22.7 \%$ $(n=15)$ presentaban 2 comorbilidades, el $16.7 \%(n=11)$ llegó a presentar hasta 4 comorbilidades y el $3 \%(n=2)$ no presentó ninguna comorbilidad (Gráfico 2).

La comorbilidad con mayor prevalencia fue la anemia, con $81.7 \%(n=49)$, seguida de la enfermedad pélvica inflamatoria con $56.7 \%(n=34)$ y la miomatosis con $30 \%(n=18)$. La infertilidad se presentó en un $18.3 \%$ $(n=11)$, la obesidad $10 \%(n=7)$ y las comorbilidades con una prevalencia menor al 10\%, fueron la ansiedad con $6.7 \%(n=4)$, la depresión, la hipertensión arterial y la patología coronaria con $5 \%(n=3)$, la diarrea se presentó en $3.3 \%(n=2)$, el colon irritable y la constipación se presentaron en $1.7 \%(n=1)$ de las pacientes. Cabe resaltar que la media del Índice de Masa Corporal encontrado en las 7 pacientes con obesidad fue de $34.3+-3.8 \mathrm{~kg} / \mathrm{m} 2$ (IC 95\%) (Tabla 2). No se presentaron casos de cáncer de ovario, infarto de miocardio, ni linfoma no Hodking en el grupo en edad fértil, pero sí en el grupo de mayores de 50 años, 1 caso de cáncer de ovario, representando el $1.5 \%$ (Tabla 2).
La anemia dividida por grupos etarios también fue la que tuvo la mayor prevalencia, tanto en el grupo de 41 a 50 años, como en el grupo de 20 a 40 años con $84.6 \%(n=22)$ y $79.4 \%(n=27)$ respectivamente. La enfermedad pélvica inflamatoria tuvo una mayor prevalencia en el grupo de 41 a 50 años con $76.9 \%$ $(n=20)$, a diferencia del grupo de 20 a 40 años con $41.2 \%(n=14)$, al igual que la miomatosis donde el grupo de 41 a 50 años presentó una prevalencia de $38.5 \%(n=10)$ y $23.5 \%(n=8)$ en el grupo de 20 a 40 años. La infertilidad tuvo una mayor prevalencia en el grupo de 20 a 40 años con $26.5 \%(n=9)$ contra $7.7 \%$ $(n=2)$ del grupo de 41 a 50 años (Tabla 2).

En el grupo de 20 a 40 años las comorbilidades con una prevalencia menor al $10 \%$ fueron la ansiedad con 5.9\% $(n=2)$, la depresión, la hipertensión, la diarrea, la obesidad y la constipación con $2.9 \%(n=1)$ cada una y en el grupo de 41 a 50 años fueron la ansiedad, depresión, infertilidad e hipertensión con $7.7 \%(n=2)$ y la diarrea y el colon irritable con $3.8 \%(n=1)$ cada una (Tabla 2).

A pesar de que el grupo de 20 a 40 años de pacientes diagnosticadas con endometriosis sea más numeroso, el grupo de 41 a 50 años presentó una mayor prevalencia de comorbilidades con 51.9\% $(n=70)$, con respecto al primero con $48.1 \%(n=65)$ de comorbilidades (Gráfico 3 ).

Dentro del número de comorbilidades presentadas por cada paciente, se registró hasta 4 comorbilidades por cada una, de este grupo las mujeres entre 20 a 40 años presentaron en 10 de estos casos enfermedad inflamatoria pélvica, en 8 anemia, en 5 miomatosis, 4 tuvieron obesidad y 3 eran infértiles. En cambio, las pacientes que presentaron 3 comorbilidades a la vez, 16 de ellas padecían anemia, 13 enfermedad inflamatoria pélvica, 7 miomatosis, 3 infertilidad y 2 obesidad y en el grupo de pacientes con 2 comorbilidades a la vez, la anemia también se presentó en la mayoría de pacientes con 11 casos, seguida por la enfermedad inflamatoria pélvica con 9 , la miomatosis con 5 casos y la infertilidad con 3 casos. La obesidad no se presentó como única comorbilidad en ninguna paciente (Tabla 3).

Dentro del número de comorbilidades presentadas por cada paciente en el grupo de 41 a 50 años se registró hasta 4 comorbilidades por cada una, de este grupo las mujeres presentaron en 5 de estos casos enfermedad inflamatoria pélvica, en 4 anemia, en 3 miomatosis y 3 tuvieron obesidad. En cambio, las pacientes que presentaron 3 comorbilidades a la vez, 11 de ellas padecían anemia, 8 enfermedad inflamatoria pélvica, 5 
miomatosis y 2 obesidad. En el grupo de pacientes con 2 comorbilidades a la vez, la enfermedad inflamatoria pélvica se presentó en 6 casos, la anemia en 5 casos y la miomatosis en 2 casos, no se presentaron casos de obesidad. Ni la miomatosis, ni la obesidad se presentaron como únicas comorbilidades en alguna paciente. (Tabla 3).

\section{DISCUSIÓN}

La media de la edad fue de 37.7 años similar al encontrado por Epstein y colaboradores, que fue de 37.4 años. Esto quiere decir que a pesar de ser un grupo pequeño se comparte la misma prevalencia en cuanto a edad de las pacientes con endometriosis que poseen alguna comorbilidad ${ }^{31}$.

El grupo de 41 a 50 años además fue el grupo que presentó mayor número de comorbilidades, siendo estas, enfermedades crónicas, a diferencia del grupo de 20 a 40 años (Tabla 2). Sin embargo, qué tan relacionadas pueden estar estas comorbilidades a la endometriosis y si constituye un factor de riesgo para las mujeres peruanas, o se trata de factores independientes.

La enfermedad inflamatoria pélvica tuvo una prevalencia del $56.7 \%$ en el grupo de pacientes estudiadas, siendo la segunda comorbilidad después de la anemia, pero en el estudio de Sen-Wen Teng, se encontró una prevalencia de hasta un 76.0\%30, cifra parecida al grupo de 41 a 50 años donde su prevalencia fue de $76.9 \%$ (Tabla 2), que puede deberse a los cambios fisiológicos próximos a la menopausia, tales como alteraciones de la flora vaginal y el $\mathrm{pH}$. Sin embargo, la menopausia no es la principal causa de enfermedad inflamatoria pélvica, por lo que sería interesante identificar el nivel de asociación entre endometriosis y enfermedad pélvica inflamatoria, así como otros factores asociados.

La prevalencia encontrada de la miomatosis uterina en el hospital María Auxiliadora fue del 30\%, cifra parecida también, al $25 \%$ encontrada en el estudio de Sen-Wen Teng y cols30. Teniendo en cuenta que la mujer peruana posee una descendencia de razas muy amplia, y el estudio de Sen-Wen Teng fue realizado en una población de mujeres asiáticas (taiwanesas), podría no ser la raza un factor de riesgo de padecer endometriosis y miomatosis a la vez.

En el caso de la infertilidad, la prevalencia encontrada fue de $18.3 \%$ en el total de pacientes diagnosticadas con endometriosis, cifra similar a la encontrada por
Sen-Weng Teng con 10.2\%30, pero mucho menor a las de Hirsch y cols donde se registra un $41 \%$ de prevalencia3 y Moradi y cols con $58.8 \% 1$.

La obesidad se presentó con una mayor prevalencia en el grupo de 41 a 50 años (19.2\%, n=5), a diferencia del grupo de 20 a 40 años $(2.9 \%, n=1)$, sin embargo, Parazzini y cols mencionan que es mucho más difícil identificar los focos endometriales en una mujer obesa, además de que ellas pueden padecer de períodos anovulatorios más prolongados, razón por la cual se explicaría su baja prevalencia encontrada25. Ascenzo y cols encontraron que el $43.9 \%$ de las pacientes que estudiaron con endometriosis sufrieron aumento de peso, cifra bastante considerable teniendo en cuenta el valor que se encontró de $10 \%(n=6)$ de pacientes con obesidad en nuestro estudio 26.

La ansiedad se presentó en $6.7 \%(n=4)$ de las pacientes y la depresión en un $5 \%(n=3)$, cifras que tal vez no reflejen el verdadero impacto en la vida de las pacientes. Moradi y cols realizaron un estudio en 35 pacientes que padecían de endometriosis, con edades similares al nuestro que referían distintos problemas sociales, físicos y psicológicos que producía la endometriosis. El $\mathbf{7 7 . 2 \%}$ de ellas mencionan que la endometriosis interfiere mucho en su vida y sólo un $22.8 \%$ refiere que el tratamiento es eficaz1. Puede que en nuestro grupo de pacientes se puedan encontrar cifras similares si tomamos en cuenta que se presentó en 11 casos junto a otras 3 comorbilidades a la vez (Tabla 3). Por lo que determinar el impacto negativo en la vida de nuestras pacientes podría aumentar a su vez los casos de ansiedad y depresión.

La patología coronaria representó el 5\% $(n=3)$ de las comorbilidades en nuestras pacientes estudiadas, cifra también similar a la encontrada por Sen-Wen Teng con 4.9\%, lo que podría guardar relación con el grupo etario donde se presentaron estos 3 casos, de 41 a 50 años30. La hipertensión se presentó en un $5 \%(n=3)$ de nuestras pacientes, por debajo de $24 \%$ mencionado por Sen-Wen Teng y cols30. Pero, al igual que la patología coronaria, no se presentó sola, sino junto a otras comorbilidades (Tabla 3).

Sólo se encontró un caso de cáncer de ovario en el grupo >50 años, lo que guardaría relación a lo mencionado por Sen-Wen Teng y cols, donde la incidencia de cáncer de ovario es de 35.81 por cada 10,000 personas al año en mayores de 50 años, a diferencia de 4.99 por cada 10,000 personas al año en menores de 30 años30. 


\section{CONCLUSIÓN}

Se encontraron un gran número de casos de las comorbilidades estudiadas, a pesar de ser un grupo pequeño de pacientes, siendo el grupo de 41 a 50 años el más afectado.

La enfermedad inflamatoria pélvica sería la patología con mayor prevalencia, seguida de la miomatosis y la infertilidad, sino fuera por la alta prevalencia de anemia en el país, que la ubica primero.

Hay que tomar en cuenta la prevalencia de ansiedad y depresión por el impacto de vida que causan, más aún si estas se presentaban junto al menos otras 2 comorbilidades.

No hay muchos estudios nacionales para poder realizar una comparación con nuestras cifras encontradas.

\section{REFERENCIAS BIBLIOGRÁFICAS}

Contribuciones de autoría: Los autores participaron en la generación, redacción y aprobación final del artículo original.

\section{Financiamiento: Autofinanciado.}

Conflicto de interés: Los autores declaran no tener conflicto de interés en la publicación de este artículo.

Recibido: 30 de octubre del 2018

Aprobado: 12 de noviembre del 2018

\section{Correspondencia: Pedro Infante Bustamante}

Dirección: Calle Juan Peña 876, San Juan de Miraflores, Lima-Perú

Celular: (+51) 945048782

Correo:pedrogaea@hotmail.com
1. Moradi M, Parker M, Sneddon A, Lopez V, Ellwood D. Impact of endometriosis on women's lives: a qualitative study. BMC Womens Health. 4 de octubre de 2014;14:123.

2. Rogers PAW, D'Hooghe TM, Fazleabas A, Giudice LC, Montgomery GW, Petraglia F, et al. Defining Future Directions for Endometriosis Research. Reprod Sci. mayo de 2013;20(5):483-99.

3. Hirsch M, Duffy JMN, Kusznir JO, Davis CJ, Plana MN, Khan KS, et al. Variation in outcome reporting in endometriosis trials: a systematic review. Am J Obstet Gynecol. abril de 2016;214(4):452-64.

4. Buggio L, Barbara G, Facchin F, Frattaruolo MP, Aimi G, Berlanda N. Selfmanagement and psychological-sexological interventions in patients with endometriosis: strategies, outcomes, and integration into clinical care. Int J Womens Health. 2 de mayo de 2017:9:281-93.

5. Machairiotis N, Stylianaki A, Dryllis G, Zarogoulidis $P$, Kouroutou $P$, Tsiamis $\mathrm{N}$, et al. Extrapelvic endometriosis: a rare entity or an under diagnosed condition? Diagn Pathol. 2 de diciembre de 2013;8:194.

6. Romero JP. Actualización sobre endometriosis. Rev Peru Ginecol Obstet. 21 de mayo de 2015;45(4):235-48.

7. Bozkurt $M$, Çil AS, Bozkurt DK. Intramuscular Abdominal Wall Endometriosis Treated by Ultrasound-Guided Ethanol Injection. Clin Med Res. diciembre de 2014;12(3-4):160-5.

8. Busard MPH, Mijatovic V, van Kuijk C, Hompes PGA, van Waesberghe JHTM. Appearance of abdominal wall endometriosis on MR imaging. Eur Radiol. mayo de 2010;20(5):1267-76.

9. Kumar S, Tiwari P, Sharma P, Goel A, Singh JP, Vijay MK, et al. Urinary tract endometriosis: Review of 19 cases. Urol Ann. 2012;4(1):6-12.

10. Molinar L, Romero P, Padilla MÁ. Endometriosis parenquimatosa pulmonar multifocal. 2011;5.

11. Ceccaroni M, Roviglione G, Rosenberg P, Pesci A, Clarizia R, Bruni F, et al. Pericardial, pleural and diaphragmatic endometriosis in association with pelvic peritoneal and bowel endometriosis: a case report and review of the literature. Videosurgery Miniinvasive Tech. junio de 2012;7(2):122-31.

12. Jain D. Perineal scar endometriosis: a comparison of two cases BMJ Case Rep [Internet]. 29 de julio de 2013 [citado 28 de abril de 2018];2013. Disponible en: https://www.ncbi.nlm.nih.gov/pmc/articles/ PMC3736109/
13. Ayala RS, Urbina JAG, Luque JLF, Medrano LD, Alarcón DC, Guerrero $\mathrm{MH}$. Endometriosis apendicular: A propósito de un caso. :2.

14. Yantiss RK, Clement PB, Young RH. Endometriosis of the intestinal tract: a study of 44 cases of a disease that may cause diverse challenges in clinical and pathologic evaluation. Am J Surg Pathol. abril de 2001;25(4):445-54.

15. Parasar P, Ozcan P, Terry KL. Endometriosis: Epidemiology, Diagnosis and Clinical Management. Curr Obstet Gynecol Rep. marzo de 2017:6(1):34-41.

16. Molecular aspects of development and regulation of endometriosis [Internet]. [citado 27 de abril de 2018]. Disponible en: https://www.ncbi. nlm.nih.gov/pmc/articles/PMC4067518/

17. Han SJ, O'Malley BW. The dynamics of nuclear receptors and nuclear receptor coregulators in the pathogenesis of endometriosis. Hum Reprod Update. julio de 2014;20(4):467-84.

18. Rahmioglu N, Macgregor S, Drong AW, Hedman ÅK, Harris HR, Randall $\mathrm{JC}$, et al. Genome-wide enrichment analysis between endometriosis and obesity-related traits reveals novel susceptibility loci. Hum Mol Genet. 15 de febrero de 2015;24(4):1185-99.

19. Grandi G, Mueller MD, Papadia A, Kocbek V, Bersinger NA, Petraglia $F$, et al. Inflammation influences steroid hormone receptors targeted by progestins in endometrial stromal cells from women with endometriosis. J Reprod Immunol. 2016;117:30-8.

20. Ahn SH, Monsanto SP, Miller C, Singh SS, Thomas R, Tayade C. Pathophysiology and Immune Dysfunction in Endometriosis. BioMed Res Int [Internet]. 2015 [citado 27 de abril de 2018];2015. Disponible en: https://www.ncbi.nlm.nih.gov/pmc/articles/PMC4515278/

21. Fassbender A, Burney RO, O DF, D'Hooghe T, Giudice L. Update on Biomarkers for the Detection of Endometriosis. BioMed Res Int [Internet]. 2015 [citado 27 de abril de 2018];2015. Disponible en: https://www.ncbi. nlm.nih.gov/pmc/articles/PMC4512573/

22. Macer ML, Taylor HS. Endometriosis and Infertility: A review of the pathogenesis and treatment of endometriosis-associated infertility. Obstet Gynecol Clin North Am. diciembre de 2012;39(4):535-49.

23. Endometriosis and infertility: a committee opinion. Fertil Steril. septiembre de 2012;98(3):591-8. 
24. Králíčková M, Vetvicka V. Endometriosis and ovarian cancer. World J Clin Oncol. 10 de diciembre de 2014;5(5):800-5.

25. Parazzini F, Esposito G, Tozzi L, Noli S, Bianchi S. Epidemiology of endometriosis and its comorbidities. Eur J Obstet Gynecol Reprod Biol. 1 de febrero de 2017;209:3-7.

26. Ascenzo B, Vergara C, P AA. Endometriosis y fertilidad [Internet]. Rev Peru Ginecol Obstet. 1996;42(1):44-5. Disponible en: http://sisbib.unmsm. edu.pe/BVRevistas/ginecologia/Vol_42N1/endometriosis_y fertilidad. htm

27. Mu F, Rich-Edwards J, Rimm EB, Spiegelman D, Missmer SA Endometriosis and Risk of Coronary Heart Disease. Circ Cardiovasc Qual Outcomes. mayo de 2016;9(3):257-64.
28. Verit FF, Yucel O. Endometriosis, Leiomyoma and Adenomyosis: the Risk of Gynecologic Malignancy. Asian Pac J Cancer Prev. 30 de octubre de 2013;14(10):5589-97.

29. Elizur SE, Lebovitz O, Weintraub AY, Eisenberg VH, Seidman DS, Goldenberg $M$, et al. Pelvic inflammatory disease in women with endometriosis is more severe than in those without. Aust N Z J Obstet Gynaecol. abril de 2014;54(2):162-5.

30. Teng S-W, Horng H-C,HoC-H,Yen M-S, Chao H-T,Wang P-H, etal.Women with endometriosis have higher comorbidities: Analysis of domestic data in Taiwan. J Chin Med Assoc. noviembre de 2016;79(11):577-82.

31. Epstein AJ, Soliman AM, Davis M, Johnson SJ, Snabes MC, Surrey ES. Changes in Healthcare Spending After Diagnosis of Comorbidities Among Endometriosis Patients: A Difference-in-Differences Analysis. Adv Ther. 2017;34(11):2491-502.

\section{Indizado en: latindex}

http://www.latindex.org/latindex/ficha?folio=14280

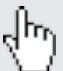

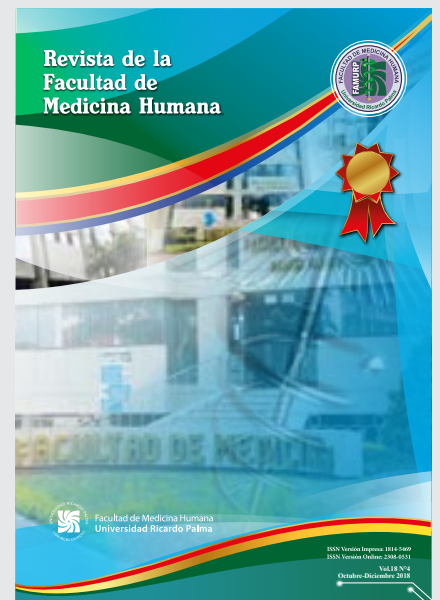

